\title{
MiLANO E IL FUMETTO, MiLANO E IL FUMETTO
}

Paolo Interdonato*

Sommario: Il fumetto, al meglio delle sue possibilità, esprime una piena coincidenza tra forma, narrazione e formato editoriale. Questa sua caratteristica lo rende uno strumento eccezionale per leggere la società che lo esprime. Una carrellata di casi esemplari ci viene da alcuni periodici nati a Milano tra la metà degli anni Quaranta e la metà degli anni Sessanta del secolo scorso: "Il Politecnico" di Elio Vittorini all'indomani della Liberazione, "Topolino" nel dopoguerra, "Tex" negli anni del Miracolo, "Diabolik" in quelli della "congiuntura" e "Linus" ad accogliere l'emergere dei giovani, dei loro bisogni e dei loro desideri.

Parole chiave: Milano. Formati di pubblicazione. Fumetto.

\section{FORME E FORMATI}

Nel suo straordinario tentativo di identificare le regole che ci consentono di dire che una lingua è impossibile, Andrea Moro (2017, p. 15) scrive:

Ci sono proprietà che scartiamo in automatico come potenziali fattori di influenza su una lingua: per esempio, non penseremmo mai che una lingua possa essere sensibile alla temperatura o alla velocità con cui la si parla; equivarrebbe a stabilire un legame ingiustificato tra proprietà formali di una lingua e le sue caratteristiche fisiche, e sarebbe insensato quanto mettere in relazione il tipo di carta usata per pubblicare un racconto e la personalità dei personaggi messi in scena.

L'esempio di Moro è così chiaro da non richiedere particolari spiegazioni: Don Quijote presente nella prima edizione del romanzo di Miguel de Cervantes Saavedra, edita da Francisco de Robles nel 1605, non è sicuramente meno Don 
Quijote del protagonista dell'edizione pubblicata nel 2005 dalla "Real Academia Española" e dalla "Asociación de Academias de la Lengua Española", per festeggiare i quattrocento anni dell'opera.

Eppure questa evidenza diventa molto più sfumata quando pensiamo al fumetto: il formato, la scelta della carta, la legatura, perfino la densità degli inchiostri cambiano la personalità dei personaggi. Non è un caso che gli autori più consapevoli definiscano accuratamente il formato di pubblicazione dei propri fumetti e lo impongano a tutti gli editori, che decidono di pubblicarne una edizione tradotta. Cito alcuni casi eclatanti: Art Spiegelman, Chris Ware e, soprattutto, Jean-Cristophe Menu.

Art Spiegelman ha scelto le dimensioni e il tipo di carta per il suo Maus (la cui prima edizione in volume è del 1986), fin dai tempi della pubblicazione del fumetto sulle pagine della rivista "Raw". Su quella testata, diretta da Spiegelman stesso e da sua moglie Françoise Mouly, Maus usciva in albi spillati che ne ospitavano un intero capitolo, con un formato e una carta diversi da quelli usati per il resto della rivista. Quel formato e quella carta sarebbero stati una costante per tutte le pubblicazioni successive di Maus ${ }^{1}$.

Chris Ware ha pubblicato parti del suo Jimmy Corrigan, The Smartest Kid On Earth nei vari formati in cui usciva Acme Novelty Library, la sua rivista d'autore edita da Fantagraphics. Il formato definitivo dell'opera è quello pubblicato nel 2000 da Pantheon Books: un libro a colori di quasi quattrocento pagine in formato orizzontale. Tutte le edizioni nazionali si adeguano a quel formato e chi legge l'edizione in brossura, in luogo di quella cartonata, non gode della meravigliosa sovraccoperta che, oltre a essere uno straordinario oggetto di design, è una mappa del racconto che arricchisce quella storia e quei personaggi ${ }^{2}$.

Ancora più manifeste sono le scelte di Jean-Cristophe Menu, autore incapace di muoversi nei formati più tradizionali del mercato francese ed editore dapprima con L'Association - collettivo di autori, il cui marchio editoriale ha a lungo diretto - e poi con L’Apocalypse. Di lui Gary Groth (2009, p. 56) dice che ha fatto "più di qualsiasi altra figura creativa per sconvolgere lo status quo e influenzare quelli che credono che il fumetto sia una forma d'arte seria". Quando gli viene chiesto cosa abbia guidato le scelte de L'Association nella definizione dei formati delle collane, Menu risponde:

Gli editori di fumetti che ci piacevano, come Futuropolis, Artefact, Humanoïdes Associés, avevano tutti i cataloghi basati sull'idea di "Collana", ognuna con un proprio design. Così, molto spontaneamente, con L'Association, abbiamo fatto lo stesso e abbiamo pubblicato molte collane. Le differenze principali tra queste collane sono i loro formati e gli assi lungo i quali si sviluppano. Ciboulette ha il formato del graphic novel. Eperluette ha le dimensioni del classico "album" francese (anche se a noi non piace usare questa parola). I Patte de Mouche sono

\footnotetext{
L'interesse di Art Spiegelman per la forma e i formati del fumetto innerva tutta la sua opera. Un'analisi dettagliata del lavoro sulla forma di Maus è contenuta in Spiegelman (2011, p. 164-234) e, in particolare, nella terza sezione della lunga intervista attorno alla quale il libro è costruito.

2 L'uso dei formati da parte di Chris Ware è analizzato con grande attenzione in Raeburn (2004) e in Groth (1999). In Bellerman (2016, p. 116), Ware afferma: "I fumetti si creano sulla pagina in un modo che non posso descrivere o capire completamente, credo sia proprio il vero vantaggio di questa forma rispetto ad ogni altra forma artistica. In altre parole, bene quando mi si presenta qualcosa a cui non avrei pensato altrimenti. Spesso queste cose finiscono per diventare la forza trainante della storia, anche se non posso prevedere se o quando questo accadrà. Posso dire che prendo molti appunti, ma solo la metà di quello che scrivo o pianifico arriva davvero a far parte della storia finale. Le storie sembra vogliano formarsi da sole, e devo prestare attenzione alla forma che stanno prendendo e seguire quella direzione, non cercare di forzare un'idea o una situazione che non trovo più sensata".
} 
albi spillati di 24 pagine molto piccoli. Mimolette è l'etichetta dei fumetti underground di 30 pagine. Côtolette ha un orientamento più letterario. Eprouvette è la collana "teorica". E se arriva un nuovo lavoro e non corrisponde a nessuno di questi formati, creiamo una nuova collana (se ci pare che questo formato possa ospitare altri lavori) o più semplicemente lo pubblichiamo come "fuori collana", ed è proprio quello che sta succedendo sempre più spesso (al punto che quei libri stanno componendo una nuova collana in cui ogni volume ha caratteristiche diverse da quelle di tutti gli altri)" (GROTH, 2009, p. 62).

Una continua ricerca del formato giusto che rende ogni fumetto un caso a sé. Il più inclassificabile tra i libri pubblicati da L'Association è Comix 2000, un volume antologico collettivo che raccoglie duemila pagine realizzate "da 324 autori, provenienti da 29 nazioni, nati tra il 1940 e il 1982” (MENU, 1999). Antologizza in modo esclusivo i fumetti in bianco e nero e privi di parole ed è l'incarnazione stessa dell'idea di editoria di Menu: "Per me perdere denaro con libri che non vuole nessuno è la definizione di ciò che significa essere un editore" (GROTH, 2009, p. 67).

Quando, alla fine del 1999, quest'opera monumentale, ideata per segnare una linea di demarcazione completamente arbitraria tra i due millenni, vede la luce, Menu, nella sua introduzione al progetto, sintetizza il senso dell'operazione:

Veniamo alle conclusioni che possiamo trarre da questa esperienza: 1) Ogni impresa completamente impossibile è tuttavia realizzabile in certe circostanze.

2) Se non l'aveste ancora notato i fumetti sono veramente un ottimo mezzo di comunicazione universale e forse il più adatto per parlare della nostra epoca. 3) Il XX secolo ha fatto bene a finire e, anche se non dipende da noi, faremo quanto sarà possibile perché il prossimo secolo si comporti un po' meglio. 4) Questo libro non è né un dizionario né un'enciclopedia perché è assolutamente parziale e soggettivo (MENU, 1999).

Un libro imponente che racchiude i frantumi della fine di un millennio per gettare uno sguardo politico, e proprio per questo parziale e soggettivo, sul futuro del fumetto. E sfogliando le pagine sottilissime di questo libro diventa evidente che quell'oggetto è un prisma attraverso il quale leggere il primo decennio del ventunesimo secolo.

Il fumetto racconta una storia, cartografandola. Per dirla con Spiegelman, è il modo per costruire i diagrammi di una storia ${ }^{3}$. Al suo meglio, stabilisce relazioni strettissime con i formati per i quali è stato pensato dai suoi autori. Quei formati diventano un modo per leggere la società che li esprime.

Un esempio straordinario ci viene da una manciata di testate nate a Milano tra il 1945 e il 1965. Poiché, come dice Menu (1999), "il fumetto è il mezzo più adatto per parlare della nostra epoca", un'analisi di quelle pubblicazioni racconta con precisione la biografia ufficiale della città meneghina e della nazione che la ospita.

3 “My dictionary defines COMIC STRIP as a 'narrative series of cartoons'. A NARRATIVE is defined as 'a story'. Most definition of STORY leave me cold. Except the one that says: 'A complete horizontal division of a building... [from Medieval Latin HISTORIA... a row of windows with pictures on them....' The word CARTOON implies humorous intent... a desire to amuse and entertain. I'm not necessarily interested in entertainment... in creating diversions. Better than CARTOON is the word DRAWINGS, or better still... DIAGRAMS!" (SPIEGELMAN, 2012, p. 2). 


\section{UNA NUOVA CULTURA}

Il primo numero del "Politecnico" esce pochi giorni dopo la fine della Seconda Guerra Mondiale, il 29 settembre 1945. E composto da quattro pagine di grande formato, lo stesso dei quotidiani. Accanto alla testata, il sottotitolo dichiara la periodicità e, soprattutto, gli intenti del giornale: "settimanale di cultura contemporanea". Il fondatore è Elio Vittorini e con lui ci sono Albe Steiner, Franco Fortini e Vittorio Pandolfi. Benché l'editore sia il torinese Einaudi, il settimanale, per volere di Vittorini, viene pensato e impaginato a Milano, in viale Tunisia 29, a metà strada tra il gasometro di Piazzale Loreto dove fu appeso il corpo di Benito Mussolini e il centro esatto della città.

Il primo numero si apre con un editoriale firmato da Vittorini $(1945$, p. 1) che dichiara una volontà radicale di trasformare una cultura che è stata incapace di fermare gli orrori e la distruzione della guerra: "Non più una cultura che consoli nelle sofferenze ma una cultura che protegga dalle sofferenze, che le combatta e le elimini".

Quel giornale sottile è il modo perfetto per raccontare i tempi difficili della ricostruzione, che si prospetta per un paese messo a dura prova dal conflitto bellico e dai bombardamenti. Per infondere ai ricostruttori una cultura che combatta ed elimini le sofferenze, "Il Politecnico" miscela sulle sue pagine parole e immagini, annullando il conflitto tra i due codici. Lo svizzero Max Huber, ricordando i momenti durante i quali impaginava il giornale con Steiner, dice:

Ciò che colpisce, guardando adesso "Politecnico", è vedere come le idee, il contenuto degli articoli, le fotografie, le didascalie e l'impaginazione fossero una cosa sola. Non c'era un "grafico" che metteva in bella forma delle colonne di piombo, dei titoli, delle illustrazioni, ma c'era un giornale che nasceva tutto insieme ${ }^{4}$.

In un giornale in cui le parole e le immagini vivono un connubio fortissimo alla ricerca dell'equilibrio perfetto, arriva presto il fumetto. Fin dal primo numero ad accompagnare gli articoli del giornale ci sono spesso sequenze di foto, usate a scopo chiaramente narrativo.

Nell'ultima pagina del secondo numero, "Il Politecnico" ospita quello che probabilmente è il primo articolo sui fumetti pubblicato da un giornale in Italia: "Il mondo a quadretti: breve storia di un'arte per i pigri” , firmato da Giuseppe Trevisani (1945, p. 4), che, pochi mesi dopo, erediterà la gestione della grafica del giornale. In tale articolo ne seguono altri dedicati a "Super-Topolino", alla "Storia illustrata della Rivoluzione Russa d'Ottobre" o al modo in cui vengono sviluppati i film d'animazione raccontati direttamente da Walt Disney (1946, p. 4).

Accanto a questa attenzione proto-teorica, "Il Politecnico", dopo aver cambiato periodicità e formato, inizia a pubblicare fumetti. Dapprima vengono pubblicate sette strisce di Popeye disegnate da Bela Zaboly (1946, p. 82-87), assistente di Elzie Crisler Segar assurto al ruolo di disegnatore principale della serie dopo la morte dell'autore. A seguire, a corredo di un articolo sul romanzo gotico, i brevissimi adattamenti a fumetti firmati da Oreste del Buono (1947, p. 40-42) di L'italiano, o il confessionale dei penitenti neri di Ann Radcliffe e di Il castello di Otranto di Horace Walpole.

4 La citazione di Huber proviene da un'intervista condotta da Roberto Leydi per "Europeo", il 5 settembre 1974, riportata in Zancan (2009, p. 89). 
Infine, negli ultimi tre numeri, "Il Politecnico" pubblica con regolarità una striscia. La scelta è esemplare: il raffinatissimo Barnaby di Crockett Johnson (1947, p. 29-30), quasi a dire che, per ottenere una nuova cultura, gli italiani hanno diritto al meglio che anche il fumetto possa dare.

\section{Dal giornale al Libretto}

Il primo numero del "Corriere dei Piccoli" esce il 27 dicembre 1908, costa 10 centesimi di lira e ha una tiratura di 80.000 copie (GADDUCCI; STEFANELLI; TAVOSANIS, 2012, p. 47) $)^{5}$. Il nuovo settimanale si rivolge ai figli della borghesia milanese mostrando forte volontà pedagogica. Il "Corriere dei Piccoli" si affianca agli altri supplementi del grande quotidiano milanese: la "Domenica del Corriere", settimanale illustrato rivolto all'intera famiglia, il cui primo numero era uscito 1'8 gennaio 1899, e il mensile la "Lettura", pubblicato da gennaio 1901. Il formato ed i contenuti del "Corriere dei Piccoli" diventano presto lo standard editoriale cui le pubblicazioni successive si adeguano (GADDUCCI; STEFANELLI; TAVOSANIS, 2012, p. 49) $)^{6}$ : tra il 1908 e il 1922 vengono fondate circa quaranta testate a fumetti destinate all'infanzia (GADDUCCI; STEFANELLI; TAVOSANIS, 2012, p. 53).

Al di fuori di questa finestra temporale, il 31 dicembre 1932 l'editore Nerbini di Firenze porta in edicola "Topolino". L'accordo sui diritti con Gabriele Emmanuel, che distribuisce le strisce di Mickey Mouse per conto dell'agenzia King Features Syndicate (BOSCHI, 2013, p. 18), arriverà solo qualche settimana do$\mathrm{po}^{7}$. Si tratta di un giornale di grande formato, composto da otto pagine e stampato in tricromia, che affianca ai personaggi disneyani la presenza di altre comic strip. "Topolino" cambia editore 1'11 agosto 1935, con il numero 137, passando da Nerbini a Mondadori, al termine di un delicato processo di avvicendamento nei diritti ${ }^{8}$.

Nel cambio di egida editoriale, il formato del periodico rimane invariato. $\mathrm{E}$ resta così fino all'aprile 1949. Alla fine dell'anno precedente, Mondadori aveva acquistato una rotativa Vomag. Quella macchina per la stampa offset permetteva di stampare i periodici da edicola con una qualità superiore a quella di tutti i concorrenti e con un costo decisamente più basso, La rotativa era stata immediatamente usata per il lancio dell'edizione italiana di "Selezione del Reader's Digest". Pochi mesi dopo si era approfittato della presenza di quella splendida macchina per produrre il nuovo "Topolino", in un formato tascabile, identico a quello di "Selezione" (BOSCHI, 2013, p. 22-23).

Il nuovo formato non modifica solo l'idea di lettura dei figli del ceto medio borghese italiano, ma trasforma radicalmente il ritmo dei fumetti e la composizione della pagina. Si definisce un modello narrativo disneyano tutto italiano, modellato su pagine con sei vignette disposte su tre strisce: questa forma diventa sia la struttura ritmica su cui cadenzare le narrazioni italiane che il letto di Procuste su cui rimontare le comic strip e le pagine dei comic book.

\footnotetext{
5 Per una disamina storica del primo periodo di "Corriere dei Piccoli" e della sua relazione con la "Domenica del Corriere", vedi Carabba (1998).

6 "Con il suo successo, il 'Corriere dei Piccoli' [...] impone presto un nuovo standard nella presentazione dei fumetti. Le tavole vengono divise in sei-otto quadri, e le storie sono raccontate mediante quartine in ottonari in rima stampate sotto ogni vignetta, con la totale eliminazione degli effetti sonori tipografici, incluse le 'nuvolette' (i balloons)".

7 Il contratto di cessione dei diritti dalla Disney a Nerbini è riprodotto in Listri (1993, p. 50-51).

8 La successione nei diritti di edizione di "Topolino" è raccontata nel dettaglio in Gadducci, Gori e Lama (2011, p. 89-99).
} 


\section{Aria di Cambiamento}

I figli della borghesia non sono i soli ad avere bisogno di intrattenimento. Al termine del secondo conflitto mondiale, in un paese sconfitto e impoverito, l'editoria sviluppa il più economico tra i formati: dal 1948 arrivano nelle edicole italiane gli albi a striscia. Sono dei fascicoli orizzontali che risolvono, su trentadue pagine, le vicende dei grandi eroi.

Anche le edizioni Audace, che nel tempo diventeranno Sergio Bonelli Editore, pubblicano fumetti in quel formato. Il 30 settembre 1948 esce "Tex", scritto da Gianluigi Bonelli e disegnato da Aurelio Galleppini, che si firma Galep.

La dura ricostruzione del Paese dopo la Guerra richiede quasi un decennio. A quel periodo di ristrettezze economiche ne segue uno florido e ricco che chiamiamo Miracolo italiano. Convenzionalmente facciamo iniziare quest'epoca di crescita e rivalsa economica e culturale in un momento preciso: domenica, 3 gennaio 1954, la RAI inizia a trasmettere dagli studi televisivi di Torino ${ }^{9}$.

Il formato di "Tex", in quel momento di straordinaria trasformazione sociale, cambia a sua volta. Le strisce vengono montate a tre per pagina su un albo di formato più grande: un quaderno dal dorso quadro sulla cui costa può comparire il nome del personaggio e il numero dell'albo.

"Tex" a striscia, a detta di Galep, era un albo adatto ai suoi tempi:

Il formato, del tipo "a striscia", rappresentava dal lato commerciale, l'ideale per quei tempi in cui i mezzi e le materie prime, compresa la carta, scarseggiavano. Inoltre era tascabile, facile da nascondersi fra le pagine di un quaderno o di un libro: vantaggio da non sottovalutare nella temperie di "proibizionismo culturale" di quel periodo, in cui il fumetto era visto alla stregua di un veicolo di corruzione dei ragazzi $[\ldots]^{10}$.

Dopo l'inizio delle trasmissioni della neonata televisione, i giovani italiani, intrisi dall'aria del Miracolo, sentono di aver diritto a un giornalino che abbia lo stesso odore. Sono stufi di storielle in ottonari e di albi "facili da nascondere". Il nuovo "Tex" è un quaderno corposo: si compone di oltre cento pagine, raccolte in una copertina di cartone e legata in una pratica brossura. Quegli albi possono essere allineati con orgoglio su una mensola e, una volta aperti, mostrano larghe pagine su cui il bianco e nero respira: l'Ariziona di Galep non è più schiacciata in una sottile striscia di carta e lo sguardo può seguire i ranger al galoppo tra vallate, montagne, altipiani e canyon.

\section{IL RE DEL TERRORE}

I lettori del nuovo "Tex" vivono sulla propria pelle le grandi trasformazioni del Miracolo. In un arco di tempo piuttosto breve, il Paese cambia radicalmente e gli italiani si ritrovano ammantati in un nuovo sistema dei bisogni e dei consumi. In un decennio il reddito nazionale netto del paese quasi raddoppia, mentre il

\footnotetext{
9 L'idea di racchiudere simbolicamente il periodo del Miracolo italiano tra l'inizio delle trasmissioni RAl e la "congiuntura", che si sviluppa tra l'estate del 1963 e quella del 1964 viene da Crainz (2000, p. 36-59). L'autore sviluppa quell'idea e quelle analisi in Crainz (2005).

10 Citazione riportata in Boschi (2017, p. 34).
} 
numero dei contadini si riduce di un terzo in favore degli impieghi nell'industria; le città vengono investite da un'ondata migratoria che sposta la residenza di decine di milioni di persone; il numero delle abitazioni costruite ogni anno raddoppia (CRAINZ, 2005, p. 87-91).

Dopo la dura ricostruzione, Milano conferma la propria posizione di città più ricca d'Italia: nel 1965 il reddito medio annuo per abitante è di 925.000 lire, quattro volte quello di Avellino, la città più povera. Il sogno di una vita più agiata, vista in televisione, è il motore di un moto migratorio dalle campagne, soprattutto meridionali, alle città, soprattutto settentrionali. Un vero e proprio esodo che cresce progressivamente: tra il 1951 e il 1961, 300.000 persone si trasferiscono a Milano; solo nel 1962 gli immigrati sono 105.448 (FOOT, 2003, p. 58).

Ai nuovi milanesi si aggiungono i molti che si trasferiscono nell'hinterland e che quotidianamente devono raggiungere la città. Tanti, come Gigi Lamera ${ }^{11}$, prendono il treno e arrivano nella stazione di Cadorna. Escono dai convogli stipati e sciamano, con passo veloce da pendolare, dalla banchine strette al piazzale antistante.

A guardare dalla finestra questa folla frettolosa che raggiunge il posto di lavoro c'è Angela Giussani. Da quando si è separata dal marito, gestisce una piccola casa editrice e sta cercando un'idea che le consenta una presenza remunerativa in edicola. L'editoria vive ancora un periodo eroico in cui alle indagini di mercato si preferisce l'osservazione diretta dei lettori. Angela cerca di capire le esigenze di un pubblico stipato su treni che sfoglia con difficoltà riviste e giornali nei vagoni accalcati.

Guardando quella folla di pendolari, immagina un albo a fumetti che possa essere infilato nella tasca della giacca senza troppi problemi. Inventa un tascabile composto da centoventi pagine con due vignette ciascuna, contenente un'unica storia la cui durata è esattamente quella di uno spostamento in treno. Il personaggio si chiama Diabolik ed è ispirato alla malvagità assoluta di Fantômas, il protagonista del popolare ciclo di romanzi scritti da Marcel Allain e Pierre Souvestre ${ }^{12}$.

Un antieroe nero e vincente, capace di mettere a segno i suoi colpi e diventare il re del terrore per una società in pieno sviluppo. Diabolik sconfigge inevitabilmente la legge e la giustizia, e il nero degli inchiostri che si posano sulla carta porosa delle pagine dell'albo è lo stesso della tuta che lo inguaina e della sua dedizione al male.

In un momento in cui pare proprio che il benessere possa toccare tutti, $\mathrm{i}$ lettori si appassionano delle vicende di "Diabolik", tifano per il ladro ferocissimo ai danni delle sue vittime ingioiellate. Pochi mesi dopo, la crescita apparentemente inarrestabile del Miracolo italiano subisce una battuta d'arresto. Tra l'estate del 1963 e quella del 1964 gli italiani iniziano a sentir parlare di "congiuntura" e Diabolik, con il suo ritmo serrato e il suo mondo nero, è l'eroe perfetto per quei tempi.

11 Protagonista di "Prendeva il treno", canzone incisa nel 1964 da Enzo Jannacci in cui il cantautore milanese registra puntualmente la trasformazione in atto: una storia d'amore frustrato che si sviluppa accanto ai binari di treno da prendere "per non essere da meno" e alla catena di montaggio.

12 La nascita di "Diabolik", le sigarette fumate da Angela Giussani guardando piazza Cadorna dalla finestra e le influenze che la porteranno a sviluppare l'idea per il suo fumetto sono raccontate nel dettaglio in Barzi (2007). 


\section{The Times They Are A-Changin' 13}

Dopo l'estate del 1964, l'economia italiana mostra chiari segni di ripresa: si può finalmente smettere di brandire il fantasma della "congiuntura". In un Paese che riprende il suo corso, è però comparso un nuovo soggetto sociale: il giovane.

L'anno della svolta è nel 1960. L'8 aprile Fernando Tambroni è a capo di un Governo monocolore democristiano che ha ottenuto la fiducia, con una maggioranza di soli tre voti al Senato, grazie all'appoggio del Movimento Sociale Italiano (Msi). Il sostegno dei neofascisti è un debito che il Governo deve pagare, concedendo Genova, città Medaglia d'Oro della Resistenza liberata il 23 aprile del 1945, come sede del sesto Congresso del Msi. Una scelta cui la città si ribella con forza. Iniziano giorni di scioperi e manifestazioni che culminano negli scontri con la polizia del 30 giugno.

I manifestanti più attivi di quei giorni non passano inosservati. I giornalisti li notano per uno strano vezzo: indossano magliette a righe orizzontali che risaltano nelle foto e nei filmati. Sono i giovani che portano in piazza capi d'abbigliamento alla moda ed esigenze nuove e diverse da quelle degli operai e dagli ex partigiani che hanno animato la manifestazione ${ }^{14}$.

A meno di tre anni dall'esordio di "Diabolik", arriva in edicola "Linus"15. È una rivista mensile completamente diversa - per formato, veste grafica e contenuti da tutti gli altri periodici a fumetti esposti in edicola e costa significativamente più di ognuno di essi (INTERDONATO, 2015, p. 46). In un mondo di giornalini, albi, libretti e quaderni, "Linus" si presenta in forma di rivista spillata stampata in ottimo bianco e nero su carta di buona qualità. Presenta sulle sue pagine una parata di fumetti scelti con grande cura. Ci sono le comic strip statunitensi come Peanuts di Charles M. Schulz, Krazy Kat di George Herriman, Li'l Abner di Al Capp, Dick Tracy di Chesfer Gould, Pogo di Walt Kelly, Barnaby di Crockett Johnson, B.C. di Johnny Hart e Wizard of Id di Hart e Brant Parker -, la striscia avventurosa inglese Jeff Hawke di Sidney Jordan e due italiani, Enzo Lunari, autore di Girighiz e Fra Salmastro da Venegono, e, soprattutto, Guido Crepax con Valentina, un fumetto che rivoluziona l'idea di costruzione della pagina nel fumetto europeo (INTERDONATO, 2015, p. 65-85).

A rendere ancora più aliena quella rivista già abbastanza eccentrica, c'è la presenza di articoli e approfondimenti sugli autori ed i personaggi dei fumetti. Sulle pagine di "Linus", si percepisce lo sguardo colto e curioso di Giovanni Gandini, strano esemplare di intellettuale milanese che ha fortemente voluto quel giornale e che ha raccolto attorno a sé un gruppo di amici dai nomi importanti: Umberto Eco, Elio Vittorini, Oreste del Buono, Franco e Bruno Cavallone, Vittorio Spinazzola, Ranieri Carano, Salvatore Gregorietti (INTERDONATO, 2015, p. 23-26).

Tra aprile 1965, quando esce il primo numero, e novembre 1971, quando Giovanni Gandini cede la proprietà e la direzione del periodico, "Linus" è il mo-

\footnotetext{
13 Titolo di una canzone di Bob Dylan pubblicata nel 1964 da Columbia Records: "Come mothers and fathers / Throughout the land / And don't criticize / What you can't understand / Your sons and your daughters / Are beyond your command / Your old road is / Rapidly agin'. / Please get out of the new one / If you can't lend your hand / For the times they are a-changin'".

14 La violenza esplosa in piazza tra giugno e luglio 1960 è al centro del romanzo Nori (2006). Per il quadro storico, vedi Crainz (2005) e Cooke (2000). Di grande interesse 1960: I ribelli, documentario del 2010 di Mimmo Colapresti, che può essere visto su YouTube (https://tinyurl.com/ya6r3can, verificato il 31/10/2018).

15 Per la storia del primo periodo di "Linus", vedi Interdonato (2015).
} 
tore della trasformazione dell'idea di fumetto in Italia e in Europa. Quello strano oggetto cartaceo è letto dai giovani (INTERDONATO, 2015, p. 191-213), che su quelle pagine trovano il sentire di tutto ciò che succede in città e nel Paese. E quando i moti della rivolta studentesca simbolicamente rappresentati dal Maggio '68 esplodono anche in Italia, ad accompagnare i giovani, c'è anche una strana rivista a fumetti che riesce a dar loro sentori di piazza e cultura altra (INTERDONATO, 2015, p. 215-235).

\section{Formato E PENSIERO UNICI}

Dagli anni Sessanta del secolo scorso ad oggi, il mercato del fumetto è cambiato radicalmente. Mentre le edicole spariscono progressivamente su tutto il territorio nazionale, i fumetti si trasformano in libri. In libreria il fumetto, grazie anche alla forza trainante dell'etichetta "graphic novel", trova nuovi lettori.

Accanto alle opere pensate per un'edizione in volume, appaiono libri dedicati ai personaggi che il fumetto seriale ha infisso con maggiore insistenza nell'immaginario dei lettori.

Ci sono volumi di grande formato, stampati su carta patinata o fattia mano di gran pregio e legati con cura in copertine cartonate, dedicati a Topolino, Tex, Diabolik o Valentina.

Scollegati dal formato di edizione per cui erano stati ideati dai loro autori, estratti dalle dimensioni delle pagine per le quali erano stati progettati, stampati su carte lontanissime da quelle originarie.

Davvero possiamo dire che "la personalità dei personaggi messi in scena" non sia stata influenzata?

\section{Milan and THE comics, Milan is THE comics}

Abstract: Comics, at their best, show a complete coincidence between form, narration and editorial format. This specific characteristic makes of comics an amazing way to understand the society expressing them. We can find interesting examples in some publications published in Milan between the mid40-s and the mid60-s of the last century: "Il Politecnico" directed by Elio Vittorini, after Italy's Liberation, "Topolino" on post-war, "Tex" in the years of the so-called "economic miracle", "Diabolik" in the time of the "critical juncture" and "Linus" that welcomed a more evident presence of young people, with their needs and wishes.

Keywords: Milan. Publishing formats. Comics.

\section{Bibliografia}

BARZI, D. Le regine del terrore: Angela e Luciana Giussani, le ragazze della Milano bene che inventarono Diabolik. Milano: Edizioni BD, 2007.

BELLERMAN, U. Un'impressione di oggettività. In: HAMELIN (a cura di). Chris Ware: Il palazzo della memoria. Scritti Disegni Interviste. Roma: Coconino Press, 2016. p. 105-119. 
BOSCHI, L. Tremila volte Topolino. In: BOSCHI, L. (a cura di). Magica Disney: 3000 volte Topolino. Napoli: Comicon, 2013. p. 17-67.

BOSCHI, L. Amici per sempre. In: Avventura magazine: Aurelio Galleppini. Milano: Sergio Bonelli Editore, 2017. p. 2-40.

CARABBA, C. Corrierino, Corrierona: la politica illustrata del Corriere della sera. Milano: Baldini \& Castoldi, 1998.

COOKE, P. Luglio 1960: Tambroni e la repressione fallita. Roma: Teti, 2000.

CRAINZ, G. L' Italia repubblicana. Firenze: Giunti, 2000.

CRAINZ, G. Storia del miracolo italiano: culture, identità, trasformazioni fra anni Cinquanta e Sessanta. Roma: Donzelli, 2005.

DEL BUONO, O. Il romanzo nero. Il Politecnico, Milano, n. 35, p. 39-42, gen./mar. 1947.

DISNEY, W. Walt Disney: la mia officina. Il Politecnico, Milano, n. 20, p. 4, feb. 1946.

FOOT, J. Milano dopo il miracolo: biografia di una città. Milano: Feltrinelli, 2003.

GADDUCCI, F.; GORI, L.; LAMA, S. Eccetto Topolino: lo scontro culturale tra fascismo e fumetti. Nicola Pesce Editore, 2011.

GADDUCCI, F.; STEFANELLI, M.; TAVOSANIS, M. Giornali per l'età dell'entusiasmo: Pubblici infantili, fantasie grafiche e rime baciate. In: BONO, G.; STEFANELLI, M. (a cura di). Fumetto! 150 anni di storie italiane. Milano: Rizzoli, 2012. p. 44-54.

GROTH, G. Understanding (Chris Ware's) Comics. The Comics Journal, Seattle, n. 200, Dec. 1997.

GROTH, G. (introduced by) Conversations. The Comics Journal, Seattle, n. 300, Nov. 2009.

INTERDONATO, P. Linus: storia di una rivoluzione nata per gioco. Milano: Rizzoli Lizard, 2015.

JOHNSON, C. Barnaby e il signor O’Malley. Il Politecnico, Milano, n. 37, p. 29-30, ott. 1947.

LISTRI, P. F. Il mondo di Nerbini: un editore nell'Italia unita. Firenze: Nerbini Editore, 1993.

MENU, J. (Coord.). Comix 2000. Paris: L’Association, 1999.

MORO, A. Le lingue impossibili. Milano: Raffaello Cortina Editore, 2017.

NORI, P. Noi la farem vendetta. Milano: Feltrinelli, 2006.

RAEBURN, D. Chris Ware. London: Laurence King Publishing, 2004.

SPIEGELMAN, A. Metamaus. New York: Pantheon Books, 2011.

SPIEGELMAN, A. Co-mix: une rétrospective de bandes dessinées, graphisme et débris divers, Paris: Flammarion, 2012.

TREVISANI, G. Il mondo a quadretti: breve storia di un'arte per i pigri. Il Politecnico, Milano, n. 2, p. 4, ott. 1945.

VITTORINI, E. Una nuova cultura. Il Politecnico, Milano, n. 1, p. 1, sett. 1945. 
ZABOLY, B. (autore non esplicitato). Una storia di Popeye. Il Politecnico, Milano, n. 31-32, p. 42-47, lug./ago. 1946.

ZANCAN, M. "Il Politecnico" e la narrativa del dopoguerra. In: ESPOSITO E. (a cura di). Il dèmone dell'anticipazione: cultura, letteratura, editoria in Elio Vittorini. Milano: Fondazione Arnoldo e Alberto Mondadori, 2009. p. 83-99.

Recebido em 11 de novembro de 2018. Aprovado em 23 de março de 2019. 